\title{
Study on Brain Tumor Classification Through MRI Images Using a Deep Convolutional Neural Network
}

\author{
Kirti Sharma, J. C. Bose University of Science and Technology, India \\ (iD) https://orcid.org/0000-0001-8472-0184 \\ Ketna Khanna, J. C. Bose University of Science and Technology, India \\ Sapna Gambhir, J. C. Bose University of Science and Technology, India \\ (iD) https://orcid.org/0000-0001-5020-8000
}

Mohit Gambhir, Ministry of Education, Faridabad, India

\begin{abstract}
Brain tumor (glioma) is one of the deadliest diseases that attacks humans, and now even men or women aged 20-30 are suffering from this disease. To cure a tumor in a person, doctors use MRI machines because the results of MRI images are proven to provide better image results than CT-scan images, but sometimes it is difficult to distinguish between the MRI images having tumors with images without tumors. However, using features of image processing techniques like scaling, contrast enhancement, and thresholding based in deep neural networks, the scheme can classify the results more appropriately and with high accuracy. In this paper, this study reveals the nitty-gritty of brain tumor (gliomas) and deep learning techniques for better inception in the field of computer vision.
\end{abstract}

\section{KEYWORDS}

Artificial Intelligence, Brain Tumor, Computer Vision, Convolutional Neural Network, Criminal Identification, Deep Learning, Glioma, MRI

\section{INTRODUCTION}

Gliomas are the most common type of primary brain tumor. This type of tumor originates from glial cells, which are neuronal support cells whose function is to help determine synaptic contact and maintain the signaling capabilities of neurons (Mayo Clinic, n.d.; Peretti, 2013a). According to (Krizhevsky et al., 2012a; Larjavaara et al., 2007) more than $50 \%$ of the 25,000 people annually suffers from glioma which is a type of brain cancer. Gliomas are classified into 4 types based on the type of cell, namely astrocytoma, ependymoma, oligodendroglioma, and unspecified glioma. The characteristics of each type of tumor vary depending upon the location and level of tumor malignancy. One of the methods used to detect glioma is radiological examination. This radiological examination is carried out by a radiologist through manual MRI medical imaging. Manual examination takes a long time despite using trained medical personnel. Brain structure, size, and tumor shape varying the level of difficulty in glioma classification, so there is a need of method that can simplify the 
classification of gliomas through medical images. Oligodendroglioma in general can be characterized by calcification, has clear borders such as round or oval, slight bleeding concentrated at one location (focal), increased contrast, tissue swelling caused by excess fluid in the body (edema) and mass effect due to compression and because of accumulation of brain fluid (Esteva, Novoa, \& Ko, 2017). Oligodendroglioma is often found in the cerebrum, in the temporal and frontal lobes (Drevelegas, 2011; Kumar \& Thulkar, 2020). Meanwhile, astrocytoma can be in any part of the brain including the cerebrum, cerebellum, and the central area of the brain, spinal cord, and brainstem (American Brain Tumor Association) (Asimellis, 2020). Complex brain structures make it difficult for radiologists to detect the shape of tumor, size, and location of the tumor. This process can be time consuming which results in increment of treatment time period of the patient. In previous research, a deep neural network has been implemented in training 129,450 clinical images, and their performances were tested by 21 dermatologists certified clinical biopsy images. Then in another study, classification of 259 patients with low and high gliomas with high accuracy: IDH1 mutation status, 94\%; Codeletion 1p / 19q, 92\%; and status methylation of MGMT promoter, $83 \%$. Each genetic category is also associated with features typical imaging (Peretti, 2013b).

This proves the role of computer systems in helping the Glioma classification process. Deep Learning technology is an algorithm which is very useful for solving various problems of human life. Powered by the enormous computational and dataset advancements, Deep Learning proved to exceed humans in visual tasks and object recognition. This algorithm improves the performance of the Convolutional Neural Network (CNN) used for recognition of objects in images (Image Processing).

\section{BACKGROUND}

Gliomas is a type of brain tumor. A Glial cell is the type of supportive cell which is present in our brain. Astrocytes, oligodendrocytes, and ependymal are the types of supportive cells. Grade is assigned to Glioma that basically indicates that how aggressive tumor is likely to be. If grade is high that means tumor is more aggressive and can grow quickly. Various types of glioma are gives below.

\subsection{Types of Brain Tumors}

In an adult human body, usually new cells will form to replace old cells or cells that are damaged. Whereas in infants and children, besides being used to replace old cells and damaged cells, the new cells are formed that are also used to complete growth in infants and children. When the old cells do not die, but the formation of new cells that are not needed by the body continues, these new cells can be called tumors (ABTA) (Asimellis, 2020). A brain tumor is a mass of cells that are not needed and develop in the brain. Based on the way they are formed, brain tumors are classified into two types, namely:

1. Primary Brain Tumors: Primary brain tumors are tumors that are formed in brain from cells and also have a tendency to remain in the brain and do not spread to other areas. Glioma is a type of brain tumor that most often occurs in primary brain tumors. Glioma is a type of brain tumor that arises from glial cells (Rajinikanth et al., 2020). Glial cells are neuron support cells whose function is to help determine synaptic contact and maintain the signaling ability of neurons (Eltahawi \& Tizhoosh, 2010). Gliomas can be classified into four types, namely: astrocytoma, ependymoma, oligodendroglioma and unspecified glioma.

2. Astrocytoma: Astrocytoma is a tumor arising from astrocyte cells. Astrocyte cells are starshaped cells that have a function to regulate the regeneration of cells that support brain tissue. Astrocytoma can appear in various parts of the brain and nervous system, which includes the cerebellum, cerebellum, central brain region, brainstem, and spinal cord. The most common early symptoms of astrocytoma are headache, seizures, memory loss, and behavior changes. 
Treatment options that can be made depend upon the type, size and location of the tumor, how far the tumor has spread, previous treatment received, and the patient's overall health (ABTA) (Asimellis, 2020). Astrocytoma is divided into two levels, namely low-grade astrocytoma (lowgrade astrocytoma) and high-grade astrocytoma (high-grade astrocytoma). Low-grade astrocytoma is usually localized and grows over a long time. Meanwhile, high-grade astrocytoma grows in fast time period and requires different treatment. Diffuse Astrocytoma: Diffuse Astrocytoma (also called Low-Grade or Grade II Astrocytoma) is divided into different types: fibrillary, gemistocytic, and protoplasmic. This tumor is classified as a grade II astrocytoma. This type of tumor tends to invade the surrounding tissue and grow at a relatively slow rate. These tumors are classified by the appearance and behavior of their constituent cells. If the tumor is accessible and can be completely removed at the time of surgery, the only additional treatment required is a follow-up scan.

3. Ependymoma: Ependymoma arises from ependymal cells that line the ventricles of the brain and the center of the spinal cord. Ependymoma is characterized by soft, grayish or red tissue that may contain mineral cysts or calcification. Symptoms experienced by ependymoma sufferers vary according to the location and size of the tumor. In infants, an increase in head size may be one of the first symptoms to appear. As the tumor develops, the patient will experience difficulties like sleeping and vomiting. The most common symptoms that older children and adults suffer from are nausea, vomiting and headaches. The first step in treating ependymoma is to remove as much of the tumor as possible. Chemotherapy can be used to remove tumors that have grown back after radiation therapy, or to delay radiation in babies and very young children. The American Brain Tumor Association (ABTA) (Asimellis, 2020) classifies Ependymoma into different types, namely:

a. Subependymoma: Subependymoma is a tumor level I which has relatively slow growth. Subependymomas usually grow near the ventricles of the brain.

b. Myxopapillary ependymoma: Myxopapillary ependymoma is a tumor grade I which has a relatively slow growth. Myxopapillary ependymoma usually grows on the lower part of the spine.

4. Oligodendroglioma: Oligodendroglioma originates from oligodendrocyte cells. Oligodendrocyte is a type of cell that forms a system whose function is to support brain tissue. Oligodendroglioma generally forms soft tissue (soft), and the tumor is grayish or pink in color. Because Oligodendroglioma growth is generally slow, Oligodendroglioma tumors often have developed for years before they can be diagnosed (ABTA) (Asimellis, 2020). Oligodendroglioma can develop anywhere in the hemisphere of the brain, although the frontal and temporal lobes are the most common locations. Oligodendroglioma located in the frontal lobe can cause weakness on one side of the body, personality or behavior changes and difficulty remembering short-term memories.

\subsection{Medical Imaging Techniques Detecting Glioma}

Imaging has an important role in evaluating patients with brain tumors. Computed Tomography (CT) and Magnetic Resonance Imaging (MRI) are the two most commonly used imaging studies which describe brain tumors. Imaging using CT and MRI has a significant impact on patient health. In improved CT and MRI techniques, the use of new imaging techniques significantly improves detection and evaluation of brain tumors. When a patient is suspected of having a brain tumor clinically, radiological evaluation is required to determine the location of the tumor, its extent and its relationship to surrounding structures. This information is very important and critical in deciding what therapy to be used by patients, such as surgery, radiation, and chemotherapy (Rajinikanth et al., 2020): 
1. Computed Tomography: Computed tomography (CT) was introduced into clinical practice in 1972 and has quickly become a very important factor in radiological diagnosis. By using CT in neuroradiology, images of the brain can be produced directly. Brain CT, which has become the procedure of choice for evaluation and diagnosis of brain tumors, has replaced invasive procedures such as pneumoencephalography or cerebral angiography (Rajinikanth et al., 2020). Improved image quality, reduced costs, and reduced number of scans to be performed have resulted in significant developments in the use of CT applications. While using CT, an increase in the characteristic differences between the lesion and surrounding normal tissue will be seen after administration of contrast agents. Lesions are areas of the brain that have been damaged by a brain tumor. Figure 1 shows an example of a CT image performed on the brain.

2. Magnetic resonance imaging (MRI): An MRI scan produces an image of the body which takes advantage of the magnetic properties of the atomic nuclei of hydrogen in water molecules found in the human body. The basic principle of MRI is to study response network in a magnetic field against radio frequency waves, where the diseased tissue reflects a different signal than normal network. T1 weighted and T2 weighted are the categories of MRI. We can differentiate these 2 types of MRI by the time of echo and repetition time while conducting it. We can classify the brain as cerebrospinal fluid (CSF), gray matter and white matter. CSF is darker in T1 weighted MRI and brighter in T2 weighted MRI(Khanna et al., 2020). MRI can provide the information details regarding the anatomy of the human body compared to that obtained using a standard $\mathrm{x}$-ray device, ultrasound, or computer tomography (CT). An example of an MRI brain image can be seen in Figure 2.

Figure 1. Example of CT Scan of the Brain (source: https://prod-images-static.radiopaedia.org/images/4170261/ c5d7c3ed6c7fe53e59c2dd902e44b9_big_gallery.jpg)

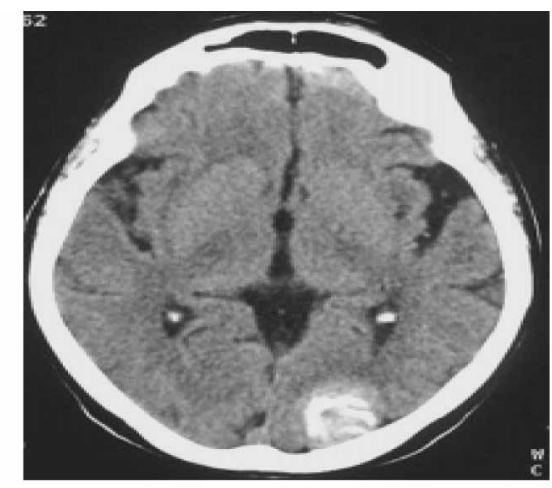

\subsection{Image Processing}

Image processing is a method used to process or manipulate digital images to produce new images (Ashley, 2020; El-Amir \& Hamdy, 2020). Image processing aims to improve image quality so that it is easily interpreted by humans or machines (in this case computers) so that it can provide new, more useful information. Image processing manipulates the image into a new image. So, input data is an image and the output is also in the form of an image, but the output image has better quality than the input image:

1. Gray scaling: Gray scaling is the process of changing a color image (RGB) into a gray image. Gray scaling is used to simplify the RGB image model which has 3 matrix layers, namely the 
Figure 2. Example of MRI in the Brain (source:https://www.kenhub.com/en/library/anatomy/normal-brain-mri)

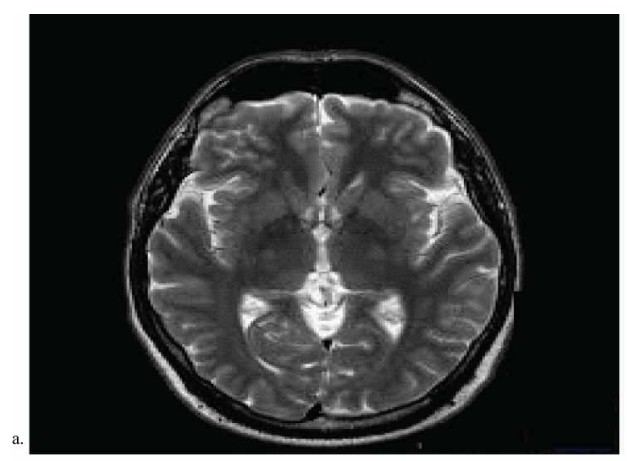

red, green, and blue matrix layers into one gray matrix layer. Gray scaling is done by multiplying each of the red, green, and blue values by a constant that amounts to 1 , as shown in equation 1 :

$I(x, y)=\alpha \cdot R+\beta \cdot G+\gamma \cdot B$

where:

$\mathrm{I}(\mathrm{x}, \mathrm{y})=$ gray scaling image pixels

$\alpha, \beta, \gamma=$ constants whose sum is 1

$\mathrm{R}=$ red value of a pixel

$\mathrm{G}=$ green value of a pixel

$\mathrm{B}=$ the blue value of a pixel

2. Cropping: Cropping functions to produce a specific part of an image by cutting unwanted areas or areas containing unnecessary information. Cropping can be used to increase focus on objects, remove unnecessary parts of the image, enlarge certain areas of the image, change the orientation of the image, and change the aspect ratio of an image. If the cropping image is used for another process, the processing time will be faster because the part that is processed is only the part that is needed.

3. Gaussian Filter: Gaussian filter functions to remove noise from the image. Noise removal is carried out in order to produce better quality images (El-Amir \& Hamdy, 2020; Pinaya et al., 2019). The filter used is Gaussian 2-D using the following equation:

$G(x, y)=\frac{1}{2 \pi \sigma^{2}} \exp \left(-\left(\frac{x^{2}+y^{2}}{2 \pi \sigma^{2}}\right)\right)$

where $\sigma$ represents the standard deviation of the distribution; The function in equation 2 is assumed to have a mean of 0 (center of distribution on the line $\mathrm{x}=0$ ). The greater the value of $\sigma$, the wider the gaussian distribution curve and the peak decreases. 
4. Contrast Stretching: Contrast Stretching is the process of improving image quality by expanding the distribution of grayscale pixels so that the probability of the appearance of the gray degree value of a pixel in the image will be evenly distributed (Putra, 2010). Calculation of contrast stretching value can be seen in equation 3 :

$$
P_{\text {out }}=\left(P_{\text {in }}-c\right) \frac{(b-a)}{(d-c)}+a o(i, j)=\frac{u(i, j)}{(d-c)}(L-1)
$$

where:

Pout $=$ grayscale pixel after being transformed

$P i n=$ grayscale pixel before transformation

$d=$ maximum value of input image pixel

$c=$ minimum value of input image pixel

$L=$ maximum grayscale value

- Image: Image is a representation (picture), similarity or imitation of an object. Image as the output of a data recording system can be optical in the form of photos, analog in the form of video signals such as images on a television monitor, or digital in nature which can be directly stored on a storage medium (de Beeck, 2019; Ilango \& Marudhachalam, 2011; Turk, 2019). Digital images can be classified into binary images, color images, and gray images (Bhattacharjee, 2020; Jain, 2019; Lee, 2020; Watson, 2020). Binary image is an image which have a special intensity where the pixel has only two values that is black and white. This value is usually encoded using a single bit which has a value of 0 or 1 on each pixel.

- Color Image: Most color images are coded with three main colors namely red, green, and blue, usually using 8 bits in every component. In a color image, each pixel requires 24 bits to encode the three components, with the value of each color component in between [ $0 \ldots 255]$.

- Gray image (Grayscale Image): Gray image is formed from a pixel channel that represents intensity, brightness and density. Pixels that represent the gray value are positive values between [0 ... 255], where the value of 0 is the minimum value of the brightness level that represents black, on the other hand 255 is the maximum value of the brightness level that describes the color white and the value between black and white represents the color gray -ash. Gray is a color where the red, green, and blue components have the same intensity.

- Computer Vision: Computer Vision is a branch of science in the field of computer that focuses and studies how machines can extract information in a static or dynamic image (Ayyadevara, 2018; Cho \& Park, 2017; Kamath et al., 2019). In contrast to the branch of Image Processing, it processes digital images or images to repair or transform digital images, while computer vision converts images into models, so that machines can recognize these images. Currently, Computer vision has been combined with many techniques, not only image processing and pattern recognition, computer vision is also often combined with existing methods in machine learning and deep learning, so that computers can not only see, but can learn from what is seen. There are many opinions about what factors cause computer vision to work well, there are at least 3 factors including computational theory, representations and algorithms, hardware implementation (Ayyadevara, 2018; Cho \& Park, 2017; Kamath et al., 2019). 


\subsection{Methodology}

\subsubsection{Deep Learning}

Deep Learning which is also known as deep structured learning or hierarchical learning is a part of Machine Learning method. Based on how the learning is divided into two parts: - Supervised Learning (e.g.: classification) and Unsupervised Learning (e.g.: pattern analysis). Deep Learning architecture is widely used in the fields of Computer Vision, speech recognition, natural language processing, audio recognition, social network filtering, machine translation, bioinformatics and others (Esteva, Kuprel, Novoa et al, 2017; Krizhevsky et al., 2012b; Munthe \& Loy, 2018; Rao et al., 2016). Deep learning can extract abstract features obtained from raw data very well (Chauhan et al., 2017). Deep Learning using a multilayered flow of non-linear processing units, each successive layer uses the output of the previous layer as input. However, deep learning requires very good hardware performance due to the large number of calculations which must be processed that also include large data.

\subsubsection{Convolutional Neural Network (CNN)}

Convolutional Neural Networks are a special type of multi-layer neural network. Almost like every other neural network, they are trained with a version of the back-propagation algorithm, the difference is in the architecture. Convolutional Neural Networks are designed to recognize visual patterns instantly of image pixels with minimal preprocessing. They can recognize patterns with extreme variability (such as handwritten characters), and with robustness to simple geometric distortions and transformations.

\subsubsection{CNN is a Trainable Architecture Consisting of Several Stages}

The input and output of each stage consists of an array which is called a feature map. Each stage consists of three layers, namely convolution, layer activation function and the pooling layer. Convolutional Neural Network architecture network can be seen in Figure 3.

Figure 3. Convolutional Neural Network Architecture

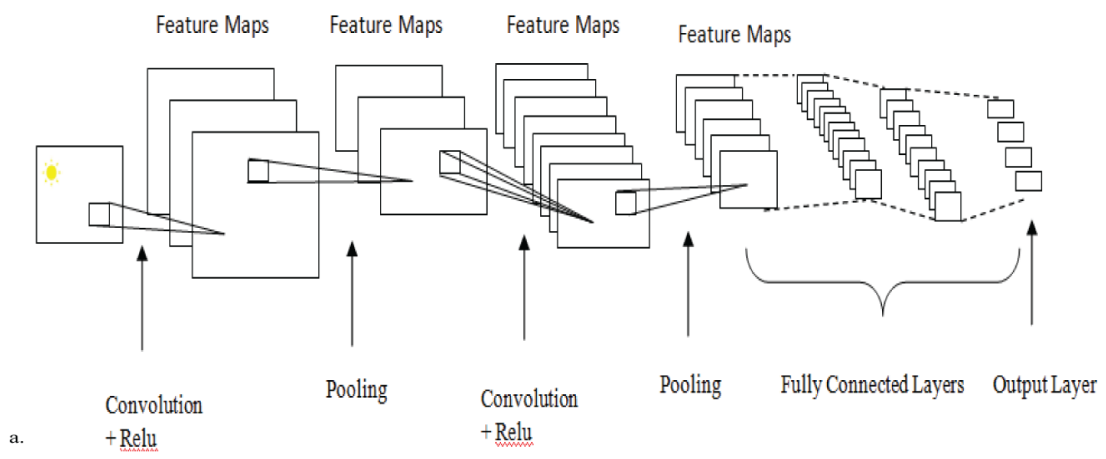

Based on Figure 3 the first stage in the CNN architecture is the convolution stage. This stage is done by using a kernel of a certain size. The calculation of the number of kernels used depends on the number of features produced. Then proceed to the activation function, usually using the ReLU (Rectifier Linear Unit) activation function, then after leaving the activation function process, then go through the pooling process. This process is repeated several times until we get a feature map that is sufficient to proceed to the fully connected neural network and from the fully connected network is the output class. Explanation of each stage of the Convolutional Neural Network: 
1. Convolution Layer: Convolution layer is part of the stage in the CNN architecture. This stage performs a convolutional operation on the output of the previous layer. This layer is the main process that underlies the CNN network architecture. Convolution is a mathematical term in which a function is applied to the output of another function repeatedly. Convolution operations are operations on two real value argument functions. This operation applies the output function as a Feature Map of the input image. Convolution operations can be written as equation 4:

$$
s(t)=\left(x^{*} t\right)(t)=\sum_{\alpha=-\infty}^{\infty} x(a) * w(t-a)
$$

Information:

$S(t)=$ Function resulting from the convolutional operation

$\mathrm{X}=$ Input

$\mathrm{W}=$ weight $($ kernel $)$

The function $\mathrm{s}(\mathrm{t})$ provides a single output in the form of a feature map. The first argument is input which is ' $x$ ' and the second argument is ' $w$ ' as kernel or filter. When viewed as a two-dimensional image input, it can be said that ' $t$ ' is a pixel and replaces it with $i$ and $j$. Therefore, the operation for convolution to input with more than one dimension can be written as in equations 5 and 6:

$$
s(i, j)=\left(K^{*} I\right)(i, j)=\sum_{\infty} \sum_{\infty} I(i-m, j-n) K(m, n)
$$

$$
s(i, j)=\left(K^{*} I\right)(i, j)=\sum_{\infty} \sum_{\infty} I(i-m, j+n) K(m, n)
$$

\section{Information:}

$s(i, j)=$ Convolutional operation result function

$\mathrm{I}=$ Input

$\mathrm{K}=$ Kernel

$\mathrm{i}, \mathrm{j}=$ pixels of the Input

$\mathrm{m}, \mathrm{n}=$ pixels from the Kernel

Equations 5 and 6 are the basic calculations in a convolutional operation, where $i$ and $j$ are the pixels of the image. The computation is cumulative and appears when $\mathrm{K}$ is the kernel, then $\mathrm{I}$ is the input and the kernel is invertible relative to the input. The determination of the output volume can also be determined from each layer with hyperparameters. The hyperparameter used in equation 7 is used to calculate the number of activated neurons in one output. Consider equation 7 :

$$
\frac{W-F+2 P}{S+1}
$$

Information: 
$\mathrm{W}=$ Image volume size

$\mathrm{F}=$ Filter size

$\mathrm{P}=$ Padding value used

$\mathrm{S}=$ Stride Size

Based on equation 7, the spatial size of the output volume can be calculated where the hyper parameters used are the volume size (W), filter (F), applied Stride (S) and the amount of zero padding used $(\mathrm{P})$. Stride is the value used to shift the filter via image input and zero padding is the value to get zeros around the image border. The first layer in the feature extraction layer is usually conv. Layers with size $5 \times 5 \times 3$. These three filters will be shifted to all parts of the image. Each shift will perform a "dot" operation between the input and the value of the filter so as to produce an output or commonly referred to as an activation map or feature map as shown in Figure 4.

\section{Figure 4. Convolution Layer}
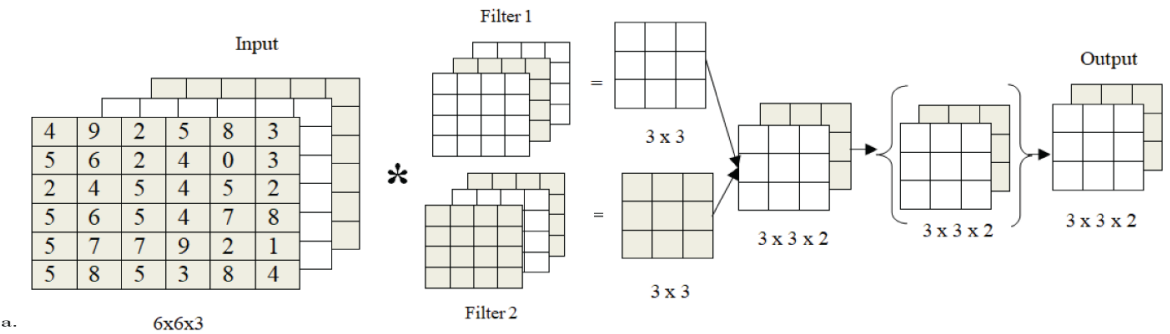

2. Pooling Operations: Pooling is a reduction in the size of the matrix using the pooling operation. Basically, the pooling layer consists of a filter with a certain size and stride which will automatically be used alternately shift in the entire feature map area. There are two types of pooling that are commonly used, namely average pooling and max pooling. The value taken at average pooling is the average value; while at max pooling is the maximum value. Pooling layers inserted between successive convolutional layers in the CNN model architecture can progressively reduce the size of the output volume on the feature map, thereby reducing the number of parameters and calculations on the network, to control overfitting. The pooling layer runs on each feature map stack and reduces its size. The form of the pooling layer generally uses a $2 \times 2$ filter that is applied in two steps and operates on each slice of the input.

Figure 5 shows the process of max pooling, the output of the pooling process is a matrix with smaller dimensions than the initial image. The pooling layer operates at each slice of the input volume depth in turn. If seen from Figure 5, the max pooling operation uses a $2 \times 2$ filter size. Put in the process the maximum value is taken from $4 \times 4$, each of the 4 numbers in the operation input is then continued to create a new output size to be $2 \times 2$.

3. Fully-Connected Layer: Fully-Connected Layer is a layer where all activation neurons from the previous layer are connected with neurons in the next layer just like in a normal neural network. The difference between the Fully-Connected layer and the regular convolution layer is that the neurons in the convolution layer are connected only to certain areas on the input, while the FullyConnected layer has neurons that are totally connected. The fully-connected process can be seen in Figure 6. 
Figure 5. Max Pooling Operation

\section{Output}
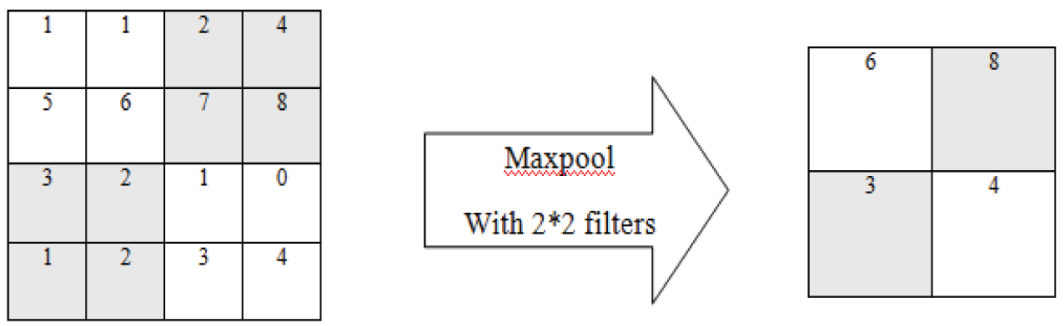

Figure 6. Fully connected layer

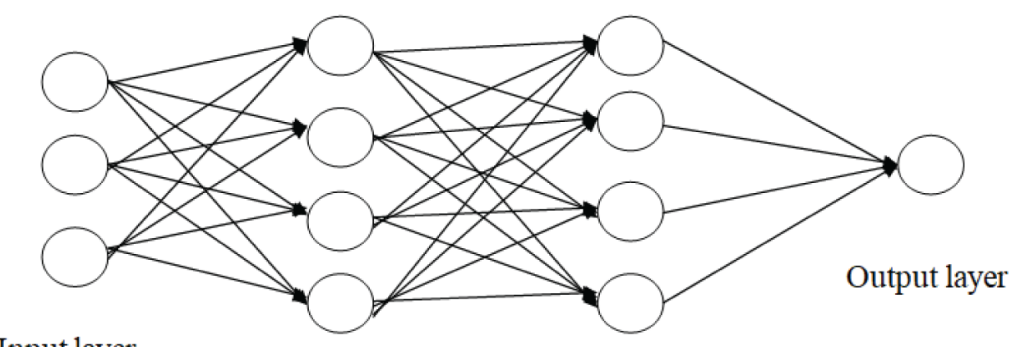

Input layer

Hidden layer $1 \quad$ Hidden layer 2

\section{LITERATURE SURVERY}

1. Hwan, et al [2017], Conducted a study by classifying Glioma into Low-grade and High-grade using Multi-modal Image Radiomics features. Tests were carried out using 220 High-Grade Glioma images and 54 Low-Grade Glioma images. This study achieved an accuracy of $88 \%$. In this MICCAI BRATs training dataset is used. With the use of radiomics features we can distinguish the high- and low-grade gliomas. For better classification, we can add additional parameters.

2. Dennis [2018], used the Deep Convolutional Neural Method Network to classify skin cancer based on the level of malignancy. Tests were carried out using images of patients suffering from skin cancer and classified based on its malignancy. This study has an accuracy of $90 \%$.

3. Krizhevsky, et al. [2012], Used Deep Convolutional Neural Network for classifying ImageNet, namely more than 15 million images high-resolution labels, comprising about 22,000 categories. Accuracy obtained using the best learning model in this study is $78.1 \%$.

4. Esteva, et al. [2017], Used the Deep Neural Network method for skin cancer classification based on skin lesion images. Tests are carried out by comparing the system performance with 21 certified dermatologists from clinical images with two use case classifications namely "keratinocyte carcinoma versus benign seborrheic keratosis" and "malignant melanoma versus benign nevi". During this research, it manages to achieve an accuracy of $93.3 \%$. 


\section{DATASET AND PREPROCESSING}

\subsection{General Architecture}

This section of scheme discusses the analysis and design in glioma classification applications through MRI images. The first stage is analysis of the data used, analysis with using several stages of image processing used, then implementation of the DCNN method in glioma classification through MRI images. However, the method used to identify brain tumors consists of: several stages. This stage begins with the collection of tumor image data brain and normal which is then used for testing data and training data the preprocessing stage is used. Midpoint filtering is used to carry out the process image pixel filtering used in CT-Scan image processing. Then the grayscale image is uniformed. The next stage is segmentation, which is the threshold to determine the process black and white through the specified threshold value. After the segmentation process is complete then perform binaryization, namely the image will be converted into a binary image. After it is entered into the classification using the Deep Convolution Neural Network. Process of all these stages will result in the identification of what the image is including brain tumors or normal.

\subsection{Dataset}

Image data used in this study is a CT-scan image obtained from https://www.kaggle.com/navoneel/ brain-mri-images-for-brain-tumor-detection/activity @ from website Kaggle.com. The CT-scan image data obtained were 300 approx. dataset images training and 253 test dataset images. Each training dataset is 95 normal and 155 brain tumors. These data will be used as testing data and training data.

\subsection{Pre-Processing}

This stage is an image processing stage that aims to produce a better image to be processed to the next stage. Pre-processing stage it consists of Midpoint filtering and grayscale.

\subsection{Midpoint Filtering}

The second stage is midpoint filtering. Where at this stage the aim is to reduce the spots from an image in order to produce a better image for the next preprocessing process; Midpoint Filter image can be seen in Figure 7.

\subsection{Grayscale}

At this stage it aims to uniform gray color in the image to be processed; In the original image visible uneven gray color. Grayscale image can be seen in Figure 8.

This stage is required to organize the pixel size of all images to be processed. The images used in this study have different pixel sizes and will be scaled for equalize it. The greater the number of pixels, the more time it will take required in the image processing process.

\subsection{Segmentation}

This stage is the stage of the process of separating one object from another object in an image into objects based on the special characteristics of an image. At this stage, the thresholding process will be carried out which is useful for separating the object and the background. Thresholding is a effective technique for image segmentation. The thresholding process is often referred to as the binaryization process. The threshold image can be seen in Figure 9.

The next processing stages used in this study are segmentation using thresholding. This process aims to produce binary image consisting of black and white. Parameters used as the thresholding value is 127 . 
Table 1. Ready references of literature review

\begin{tabular}{|c|c|c|c|}
\hline S.no. & Researcher/Year & Research Title & Information \\
\hline 1. & $\begin{array}{l}\text { H. Cho and H. Park } \\
\text { (2017) }\end{array}$ & $\begin{array}{l}\text { Classification of low-grade and high- } \\
\text { grade glioma using multi-modal image } \\
\text { radiomics features }\end{array}$ & $\begin{array}{l}\text { This study uses the Multi-modal Image Radiomics } \\
\text { features method to classify Glioma into Low-grade and } \\
\text { High-grade }\end{array}$ \\
\hline 2. & $\begin{array}{l}\text { Alex Krizhevsky, Ilya } \\
\text { Sutskever, Geoffrey E. } \\
\text { Hinton (2012) }\end{array}$ & $\begin{array}{l}\text { ImageNet Classification with Deep } \\
\text { Convolutional Neural Networks }\end{array}$ & $\begin{array}{l}\text { In this study, a Deep Convolutional Neural Network } \\
\text { was used to classify ImageNet, which is more than } 15 \\
\text { million labeled high-resolution images, consisting of } \\
\text { about } 22,000 \text { categories. The learning accuracy of the } \\
\text { best model obtained in this study was } 78.1 \% \text {. }\end{array}$ \\
\hline 3. & $\begin{array}{l}\text { Esteva, Andre \& Kuprel, } \\
\text { Brett \& Novoa, Roberto } \\
\text { \& Ko, Justin \& Swetter, } \\
\text { Susan \& Blau, Helen \& } \\
\text { Thrun, Sebastian. (2017) }\end{array}$ & $\begin{array}{l}\text { Dermatologist-level classification of } \\
\text { skin cancer with deep neural networks }\end{array}$ & $\begin{array}{l}\text { Using the Deep Neural Network method for } \\
\text { classification of skin cancer based on skin lesion } \\
\text { images. This study managed to achieve an accuracy } \\
\text { of } 93.3 \% \text {. }\end{array}$ \\
\hline 4. & $\begin{array}{l}\text { Prajwal Rao, Nishal } \\
\text { Ancelette Pereira dan } \\
\text { Raghuram Srinivasan } \\
\text { (2016) }\end{array}$ & $\begin{array}{l}\text { Convolutional Neural Network for } \\
\text { Lung Cancer Screening in Computed } \\
\text { Tomography (CT) Scans }\end{array}$ & $\begin{array}{l}\text { For Lung Cancer detection, Data Science Bowl } 2017 \\
\text { Kaggle competition data is used. With increasing } \\
\text { patients of Lung Cancer every year, it is important } \\
\text { to detect Lung Cancer so as to give proper medical } \\
\text { treatments. A 3D Convolutional Neural Network } \\
\text { (CNN) is used to detect nodules using these cubes. } \\
\text { The model achieves precision and recall of } 89.24 \% \text { and } \\
82.17 \% \text { respectively. }\end{array}$ \\
\hline 5. & $\begin{array}{l}\text { S. Chauhan, A. More, R. } \\
\text { Uikey, P. Malviya and A. } \\
\text { Moghe (2017) }\end{array}$ & $\begin{array}{l}\text { Brain tumor detection and } \\
\text { classification of MRI images using } \\
\text { image and data mining }\end{array}$ & $\begin{array}{l}\text { All the extracted features are stored in a transactional } \\
\text { database to which IBkLG classifier (Instance-based } \\
\text { K-Nearest using Log and Gaussian weight Kernels) } \\
\text { has been applied using WEKA } 3.9 \text { tool, to classify } \\
\text { the tumor into normal benign or malignant. The } \\
\text { classification accuracy is observed found to be } 86.6 \% \text {. }\end{array}$ \\
\hline 6. & Karuppathal, R.. (2014) & $\begin{array}{l}\text { Brain tumor detection and } \\
\text { classification of normal, abnormal in } \\
\text { MRI images. }\end{array}$ & $\begin{array}{l}\text { Detection of the brain tumor using Image Binary } \\
\text { Operations. Segmented is classified using SVM } \\
\text { classifier according to the feature values extracted } \\
\text { from the segmented image to find performance of } \\
\text { proposed approach. }\end{array}$ \\
\hline 7. & $\begin{array}{l}\text { Kesur, Sweta \& Rathod, } \\
\text { Prajakta. (2018) }\end{array}$ & $\begin{array}{l}\text { Enhanced Brain Tumor Detection on } \\
\text { MRI images }\end{array}$ & $\begin{array}{l}\text { The eesearchers proposed two algorithms which } \\
\text { are implemented in order to detect the exact tumor } \\
\text { part and location by first applying novel filtration } \\
\text { technique to remove noise from an image and then by } \\
\text { applying EM (Expectation Maximisation) algorithm } \\
\text { which extracts tumor part, further improved results is } \\
\text { obtained through Watershed Technique that uses image } \\
\text { segmentation and morphological operation concepts. }\end{array}$ \\
\hline 8. & $\begin{array}{l}\text { Sharma, Niharika } \\
\text { \& Mishra, Prakhar } \\
\text { \& Sadhana, K.S. \& } \\
\text { Nithyakani, P.. (2018) }\end{array}$ & $\begin{array}{l}\text { Segmenting and classifying MRI } \\
\text { images for brain tumors using CNN }\end{array}$ & $\begin{array}{l}\text { The researchers proposed two methodologies which } \\
\text { can be broadly categorized 1.General Probabilistic } \\
\text { Methods- Probabilistic methods are a remarkable } \\
\text { method to establish the validity of combinatorial } \\
\text { entities with distinct characteristics. Although the } \\
\text { basis of their existence lies in probability, they are } \\
\text { not bounded by it and can be used to solve and } \\
\text { evaluate theorems across different branches of } \\
\text { Mathematics.2.Discriminative Approaches- They are } \\
\text { also sometimes referred to as Conditional Models. } \\
\text { We utilise CNN's for faster and accurate processing } \\
\text { of the data }\end{array}$ \\
\hline
\end{tabular}


Figure 7. Midpoint Filter Image (source: https://miro.medium.com/max/3000/1*H-Rc2oZLOQdZkUselTPiig.png)

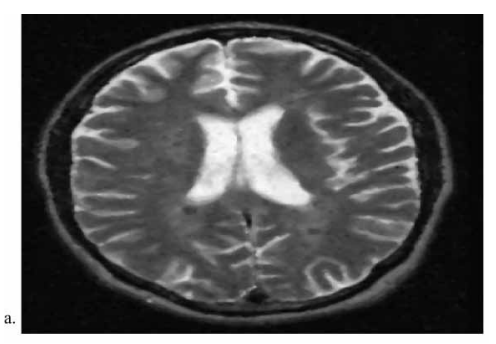

Figure 8. Grayscale Image (source: https://www.researchgate.net/profile/Abdul-Salam-Shah/publication/312023421/figure/fig3/ AS:445956740128770@1483335780108/A-Grey-scale-MRI-Image-before-the-Pre-Processing-B-Grey-Scale-MRI-Image-after-Pre.png)

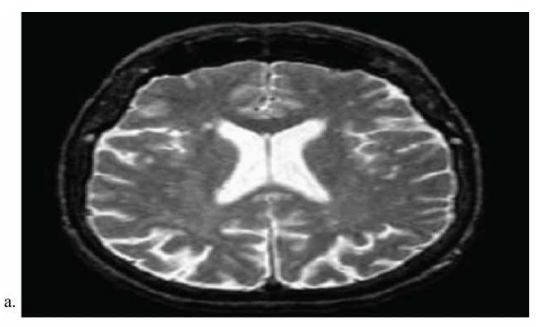

Figure 9. Threshold Image (source:https://www.researchgate.net/profile/Sriramakrishnan_Padmanaban/publication/318952747/ figure/fig7/AS:668534183047172@1536402378373/Simple-thresholding-on-MRl-a-Sample-Image-b-Threshold-image-T-14-128.png)

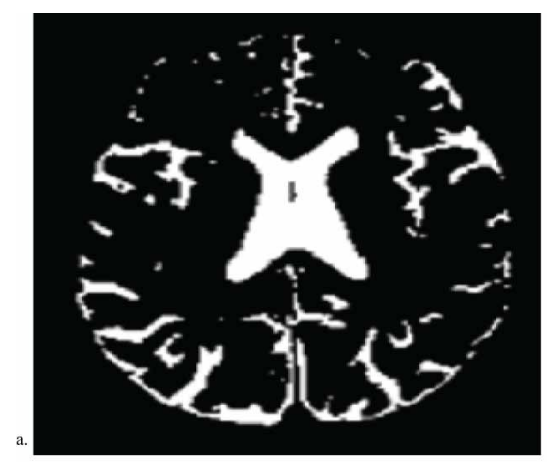

\section{IMPLEMENTATION AND RESULT}

\subsection{Training Process}

Training is the stage of implementing the DCNN model that has been created. At this stage, all images that have passed preprocessing will be input on the input layer. Every image that is entered as input will be entered into convolutional layer. The first stage is carried out in the training process in this study is the value of input weight and bias randomly. Number of neurons on input the layer is adjusted according to the parameters received from the dataset used. The second stage is the calculation of the hidden layer output matrix, which is the result of processing from the input that has been received by neurons on hidden layer of neurons in the input layer. The result of this process is a matrix represents 
the weight of each neuron in the output layer. The results of the training process will be stored in a mode with the extension based on MobileNetV2 so that it can be used in the testing process.

\subsection{Model Compiling and Training}

At this stage, the DCNN model was created. Among them determine the number hidden layer, number of neurons, activation function, optimizer loss, batch size and epochs.

\subsubsection{Determination of the Hidden Layer}

The optimal number of hidden layers used in this study is obtained by how to trial and error. In this study, the number of hidden layers used is 2 to 87 .

\subsubsection{Determination of the Number of Neurons}

There is no specific calculation in determining the number of neurons in the hidden layer.

In this study, the authors used 13 neurons in the fully-connected layer or in layer 3.

\subsubsection{Determination of the Activation Function}

The calculation results between input, weight and bias will be calculated again with the equation from the activation function to get the output of each layer. In this research, the author uses the relu activation function in the convolutional layer and the activation function Softmax on the output layer to get a result which is categorical data. Systematically, the relu activation function can be seen in equation 8:

$$
\mathrm{f}(\mathrm{x})=\max (0, \mathrm{x})
$$

where $\mathrm{z}$ is the calculation result in the layer; The Softmax function can be seen from equation 22. Therefore, Softmax classifier is a generalization for several classes. Softmax classifier gives a slightly more intuitive output (probability of that class normalized) and also has a probabilistic interpretation that will be straightforward. Softmax also delivers results that are more intuitive and have better probabilistic interpretation results compared to other classification algorithms. Result from label existing, will be taken a value vector which has real value and converting it to a vector with a value between zero and one. If all results add up will be worth one as:

$$
\sigma(z)=\frac{e^{z}}{\sum_{k=1}^{k} e^{2} k}
$$

\subsection{Optimizer Determination and Testing}

The optimization algorithm aims to find the optimal weight, minimize errors and maximize accuracy. During the training process, the parameters (weights) of the model are changed to try and minimize the function losses, in order to be able to predict as accurately as possible. But how right, when, and how many changes it still can't sure, then this is where the optimizer comes in. They put together functions losses and model parameters by updating the model in response the output of the loss function. In simpler terms, an optimizer shapes the model we've got into the most accurate form with take advantage of its weight. On research in this case, the optimizer used is Adam. Adam is optimization algorithms that can be used update the weight network iteratively based on training data. Adam's algorithm is popular on deep learning field because it achieves good results fast. The formulation composition for Adam is as under: 


$$
\begin{aligned}
& g_{t}=\nabla_{\theta_{t-1}} L\left(\theta_{t-1}\right) \\
& m_{t}=(1-\beta) g_{t}+\beta m_{t-1} \\
& \hat{m}_{t}=\frac{m_{t}}{1-\beta_{t}} \\
& \hat{v}_{t}=\frac{v_{t}}{1-Y_{t}} \\
& \theta_{t-1}=\theta_{t}-\alpha \frac{m_{t}}{\sqrt{\hat{v}_{t}}+\varepsilon}
\end{aligned}
$$

The batch size is used to determine the number of observations made before make changes in weight, which is determined relative to the specification's computer. In this study the authors used the default batch size of 50 as mentioned in Figure 10.

\subsection{Confusion Matrix}

Determining whether or not the performance of a classification model can be seen from the performance measurement parameters, namely the level of accuracy, recall, and precision:

1. Accuracy, the percentage of classifier accuracy in classifying test data correctly.

2. The error rate (misclassification rate) of the classifier with accuracy.

3. Precision is a measure of accuracy for knowing the percentage tuples that are labeled positive and completely positive.

4. The recall or true positive rate is the portion of the positive tuple correctly classified.

5. An alternative to combine precision and recall into one measure is by the f-measure approach which is also known as f1-score. The F-measure measures the accuracy and robustness of a classifier with the harmonic average of its precision and recall values. To calculate these factors a matrix is needed commonly called confusion matrix. One of the most commonly used confusion matrix in measurement can be seen in Figure 11.

\subsection{Classification and Results}

Apart from testing a single image, the system can also test the accuracy of the system which includes a group of grouped glioma brain cancer images and has not been trained by the system. The results of the system testing will output the entire image classification results based on model developed by DCNN, and ranked in order of highest accuracy as in Figure 12. 


\section{Figure 10. Loss and Accuracy Formation using Epochs}

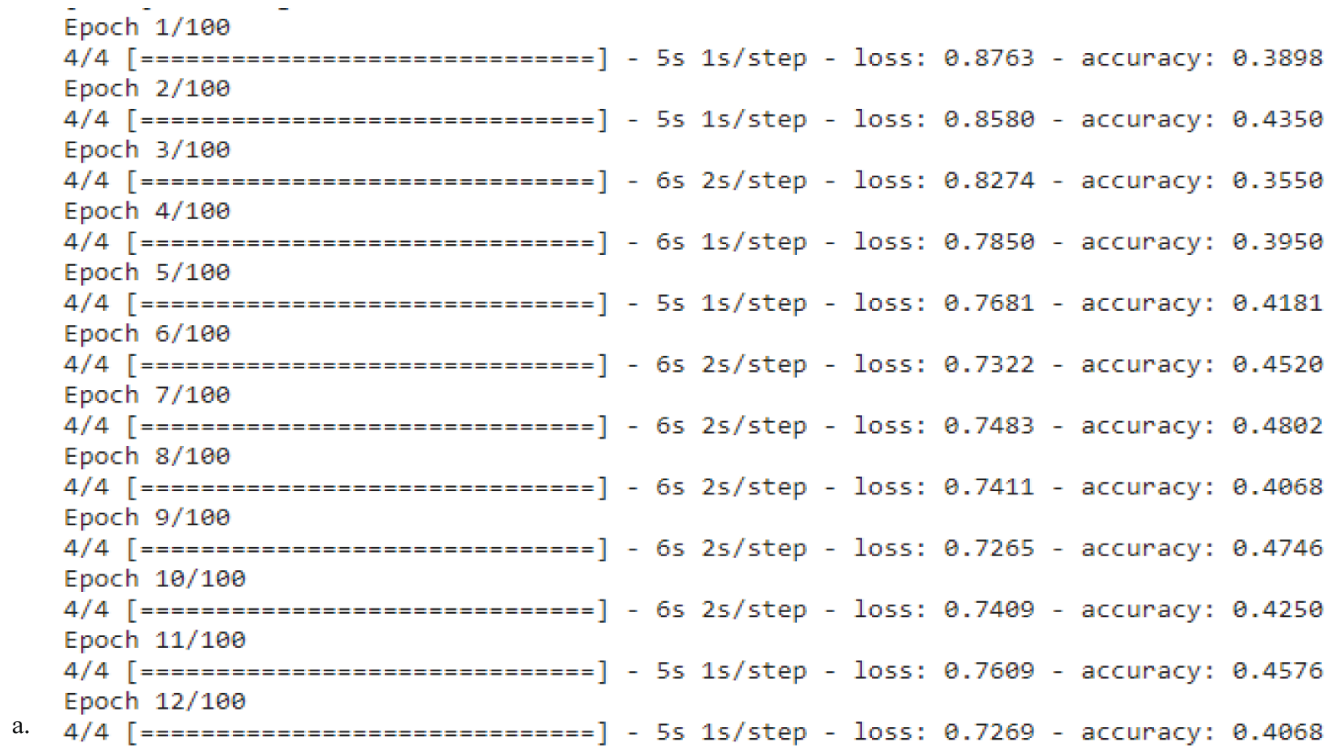

Figure 11. Confusion Matrix with Precision Recall, F1 Score and Accuracy using DCNN

[INFO] evaluating network...

precision recall f1-score support

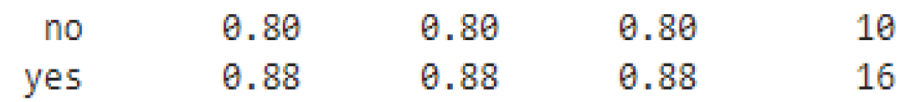

accuracy

$0.85 \quad 26$

$\begin{array}{lllll}\text { macro avg } & 0.84 & 0.84 & 0.84 & 26\end{array}$

$\begin{array}{lllll}\text { weighted avg } & 0.85 & 0.85 & 0.85 & 26\end{array}$

[INF0] saving mask detector model...

a. File Saved 
Figure 12. Result Classification using DCNN for Glioma Brain Cancer Images

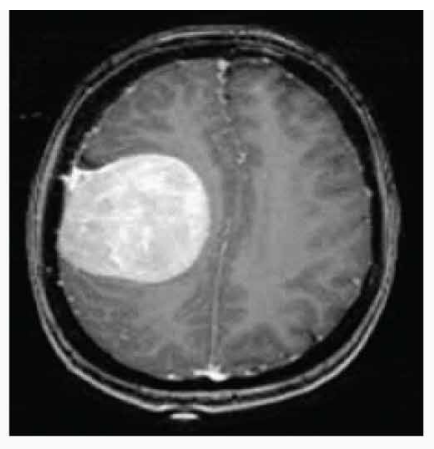

Tumor detected, with probabilty of $83.38 \%$

\section{CONCLUSION}

The conclusion that can be drawn is based on this study is that with the use of DCNN method classification of Glioma through MRI images is quite good. Using a good and appropriate feature extraction method so that the feature value from the image to be obtained to be better like scaling, contrast enhancement and thresh-holding is a combination of good image processing as input in the process glioma classification via MRI images. In the image processing process the study reveals that, the threshold used is not suitable and will affect accuracy system. In future, we can use more images to train the data in deep learning, so that data testing will result in better accuracy again. 


\section{REFERENCES}

Ashley, K. (2020). Deep Computer Vision. 10.1007/978-1-4842-5772-2_6

Asimellis, G. (2020). Imaging Definitions. 10.1117/3.2506310.ch3

Ayyadevara, V. (2018). Convolutional. Neural Networks, 179-215. Advance online publication. doi:10.1007/9781-4842-3564-5_9

Bhattacharjee, J. (2020). Computer Vision. 10.1007/978-1-4842-5121-8_6

Bousquet, N., Guedj, B., Chesneau, C., Navarro, F., \& El Kolei, S. (2018). L'apprentissage profond. Academic Press.

Chauhan, S., More, A., Uikey, R., Malviya, P., \& Moghe, A. (2017). Brain tumor detection and classification in MRI images using image and data mining. 10.1109/RISE.2017.8378158

Chityala, R., \& Pudipeddi, S. (2020). Convolutional. Neural Networks, 265-273. Advance online publication. doi:10.1201/9780429243370-12

Cho, H-h., \& Park, H. (2017). Classification of low-grade and high-grade glioma using multi-modal image radiomics features. 10.1109/EMBC.2017.8037508

de Beeck, O. (2019). Image Processing. doi:10.1017/9781316847916.009

Drevelegas, A. (Ed.). (2011). Imaging of brain tumor with histological correlations (2nd ed.). Springer. doi:10.1007/978-3-540-87650-2

El-Amir, H., \& Hamdy, M. (2020). Convolutional. Neural Networks, 367-413. Advance online publication. doi:10.1007/978-1-4842-5349-6_11

Eltahawi, O., \& Tizhoosh, , H. (2010). Image thresholding using neural network. Proceedings of the 2010 10th International Conference on Intelligent Systems Design and Applications, ISDA'10, 1159-1164. doi:10.1109/ ISDA.2010.5687030

Esteva, A., Kuprel, B., Novoa, R. A., Ko, J., Swetter, S. M., Blau, H. M., \& Thrun, S. (2017). Dermatologist-level classification of skin cancer with deep neural networks. Nature, 542(7639), 115-118. doi:10.1038/nature21056 PMID:28117445

Esteva, A. K., Novoa, B., \& Ko, J. S. (2017). Dermatologist-level classification of skin cancer with deep neural networks. https://cs.stanford.edu/people/esteva/nature10.1038/nature21056

Ilango, G., \& Marudhachalam, R. (2011). New hybrid filtering techniques for removal of Gaussian noise from medical images. ARPN J of Engineering and App Sci., 6.

Jain, A. (2019). Computer Vision. 10.4324/9780429042522-10

Kamath, U., Liu, J., \& Whitaker, J. (2019). Convolutional. Neural Networks, 263-314. Advance online publication. doi:10.1007/978-3-030-14596-5_6

Khanna, Gambhir, \& Gambhir. (2020). Current Challenges in detection of Parkinson's disease. Journal of Critical Reviews, 7.

Krizhevsky, A., Sutskever, I., \& Hinton, G. E. (2012a). ImageNet Classification with Deep Convolutional Neural Networks. Advances In Neural Information Processing Systems, 1-9.

Krizhevsky, A., Sutskever, I., \& Hinton, G. E. (2012b). ImageNet Classification with Deep Convolutional Neural Networks. https://papers.nips.cc/book/advances-in-neural-information-processing-systems-25-2012

Kumar, M., \& Thulkar, S. (2020). Imaging. Advance online publication. doi:10.1007/978-981-15-6499-4_5

Larjavaara, S., Mäntylä, R., Salminen, T., Haapasalo, H., Raitanen, J., Jääskeläinen, J., \& Auvinen, A. (2007). Incidence of gliomas by anatomic location. Neuro-Oncology, 9(3), 319-325. doi:10.1215/15228517-2007-016 PMID:17522333 
LeCun, Y., Bengio, Y., \& Hinton, G. (2015). Deep Learning. Nature, 521(7553), 436-444. doi:10.1038/ nature14539 PMID:26017442

Lee, R. (2020). Computer Vision. 10.1007/978-981-15-7695-9_5

Mayo Clinic. (n.d.). Glioma. https://www.mayoclinic.org/diseases-conditions/glioma/symptoms-causes/syc20350251

Munthe, D., \& Loy, T. (2018). Classification of skin cancer based on grade cancer malignancy using a Deep Convolutional Neural Network Field. University of Northern Sumatra.

Peretti, P. (2013a). Imaging in oligodendroglioma. International Journal of Biomedical Imaging. Advance online publication. doi:10.1155/2017/9749108

Peretti, P. (2013b). Imaging in oligodendroglioma. Medscape.

Pinaya, W., Vieira, S., Garcia-Dias, R., \& Mechelli, A. (2019). Convolutional neural networks. 10.1016/B9780-12-815739-8.00010-9

Rajinikanth, V., Raja, N., \& Dey, N. (2020). A Beginner's Guide to Multi-Level Image Thresholding. Academic Press.

Rao, P., Fereira, N., \& Srinivasan, R. (2016). Convolutional neural networks for lung cancer screening in computed tomography (CT) scans. 10.1109/IC3I.2016.7918014

SandersW. (2019). Image Processing. 10.1201/9780429266553-9

TurkI. (2019). Image Processing. 10.1007/978-1-4842-5281-9_10

Watson, S. (2020). Deep Learning. 10.1201/9780429398292-9

Ketna Khanna is currently pursuing Ph.D. in Computer Engineering from J.C. Bose University of Science and Technology, YMCA, Faridabad. She is Master of Technology in Computer Engineering and Bachelor of Technology in Computer Science and Engineering. Her main research area focuses on Machine Learning, Data Mining and Software Testing. 